\title{
EL PAPEL DE LA ENCINA EN LOS ESPACIOS ADEHESADOS
}

\author{
Ana Beatriz MATEOS RODRÍGUEZ \\ Universidad de Extremadura
}

\section{DEFINICIÓN DEL CONCEPTO DE DEHESA.}

Las definiciones del término dehesa son muchas y muy diversas, lo cual contribuye a percibir el concepto como confuso y difícil de precisar (LLORENTE PINTO, 1985). El problema reside, según el mismo autor, en establecer el porcentaje de monte que debe tener una explotación para ser considerada dehesa. A pesar de que sea una explotación dedicada fundamentalmente a la ganadería, puede calificarse como dehesa a "aquellas explotaciones en las que más del $50 \%$ de su terreno se dedica permanentemente al pastizal y al monte".

CAMPOS PALACín (1992) la define como "un sistema agroforestal cuyos componentes leñosos, pascícolas, ganaderos y agrícolas interactúan beneficiosamente en términos económicos y ecológicos en determinadas circunstancias de gestión.

En la Ley 1/1986, de 2 de mayo, sobre la Dehesa en Extremadura, encontramos una definición del término basada simplemente en su superficie. Así, es considerada dehesa "toda finca rústica de más de 100 ha susceptible de aprovechamiento ganadero en régimen extensivo".

Si consideramos la definición dada por la SOCIEDAD ESPAÑOLA PARA EL ESTUDIO DE LOS PASTOS (2001), dehesa es "una superficie con árboles más o menos dispersos y un estrato herbáceo bien desarrollado, en la que ha siso eliminado, en gran parte, el arbustivo. Es de origen agrícola (tierras labradas con rotaciones largas) y ganadero. Su producción principal es la ganadería extensiva o semiextensiva, que suele aprovechar no sólo los pastos herbáceos, sino también el ramón y los frutos del arbolado".

Así, una definición más completa de dehesa sería la de un paisaje seminatural, resultado de la acción antrópica, constituido por un arbolado disperso, fundamentalmente de quercíneas, y con un aprovechamiento extensivo agrosilvopastoril condicionado por unos factores fisicos limitantes. 


\section{ORIGEN Y GÉNESIS.}

El término dehesa se deriva del latín deffesa que, en un principio, era entendida como "aquella porción de tierra, cercada o acotada, de dominio privado, de algún municipio o de alguna persona física o particular". Hasta el año 924 no se tienen noticias de que aparezca la voz dehesa en castellano según el diccionario de Corominas, aunque con anterioridad nos encontramos en las Leyes visigodas el término referido al acotamiento de fincas, el llamado pratum defensum, (HERNÁNDEZ DÍAZ-AMBRONA, 1995).

Tras la Reconquista, se asiste a la verdadera aparición de las dehesas. La distribución del territorio recién conquistado entre las Órdenes Militares, los Señoríos y los Concejos de Realengo impondrá peculiares formas de disfrute de la propiedad de la tierra. Ésta no era ejercida de manera plena, sino que se distinguía en función de sus aprovechamientos, el suelo y el vuelo, es decir, los pastos, la montanera, la leña, la madera, etc. Para el disfrute de unos pastizales que se desarrollaban plenamente durante el invierno gracias a la suavidad del clima, se crearon las Cañadas Reales, que facilitaron la llegada de grandes rebaños de ovejas merinas. Esto originó un conflicto entre los trashumantes meseteños y los habitantes de los principales Concejos de la región, los cuales pondrían trabas a los abusos de los pastores solicitando al Rey el permiso para acotar y cerrar sus fincas, lo que llamaron defendere de los abusos trashumantes.

La nueva figura surgida así supone el mantenimiento de la explotación tradicional del pastizal-encinar principalmente por ganado de cerda, aunque dio pie a la aparición de los primeros rebaños merinos. No será hasta entrado el siglo XIX cuando comiencen a unificarse las distintas titularidades que recaían sobre las dehesas. Con motivo de la desamortización de los bienes públicos, es cuando aparecen las grandes propiedades personales. Si bien, en numerosos casos, la tradicional división de la propiedad permitió la conservación de las dehesas de encinar-pastizal prácticamente intactas hasta nuestros días, evitando así la desaparición del bosque mediterráneo adehesado.

A mediados del siglo XX, se produce la casi desaparición del cerdo ibérico debido a la peste porcina africana. Esto llevó a la reducción de la principal fuente de ingresos de los sistemas adehesados, la representada por la bellota, dando lugar a una fase de abandono de la producción forestal a favor de la agrícola (cultivos de secano y regadío), responsable de la tala y el aclarado abusivo del encinar. A ello debe unirse el fuerte proceso migratorio sufrido por Extremadura desde los años 50, que sumió a la región en una profunda despoblación del medio rural, lo que se tradujo en un abandono de muchas estructuras de explotación tradicional; la gran repoblación forestal que desde los años 60 se ha venido realizando en favor de especies alóctonas (pinos y eucaliptus fundamen- 
talmente) y en detrimento de nuestros bosques de quercíneas; y la multiplicación de los incendios forestales, traen como consecuencia que las pérdidas del arbolado de esta especie se incremente considerablemente.

Se calcula que entre 1960 y 1980 se han perdido en Extremadura más de 8 millones de pies de quercíneas (LECO BERROCAL, 1996).

El sistema adehesado tiene su origen en la acción del hombre como agente modelador del bosque mediterráneo original (BRAVO OVIEDO, 1989). Así, Elena ROSELLÓ (1986) establece seis etapas en el proceso de degradación del bosque mediterráneo por parte de la actividad humana:

I. Bosque esclerófilo mediterráneo sin estratificación. El bosque mediterráneo aparece como una masa cerrada y de difícil acceso, con todos los estratos o portes vegetativos: arbóreo, arbustivo, subarbustivo, herbáceo y subherbáceo. Sería la fisionomía que tendrían las dehesas abandonadas, en las que se produciría una regeneración del monte mediterráneo de tipo esclerófilo.

II. Etapa de pre-dehesa. Inicio de estratificación debido a operaciones culturales como la roza y poda del estrato arbustivo y subarbustivo.

III. Dehesa como monte hueco u oquedal. Etapa donde quedan perfectamente definidos los dos estratos -suelo y vuelo- sobre los que se apoya este sistema. Durante esta fase se ven favorecidos los pastos y su progresiva extensión. Es la verdadera fisionomía del ecosistema. Tanto en esta etapa como en la siguiente, la importancia de la ganadería extensiva (bovina, ovina y porcina) es un pilar esencial en el mantenimiento del ecosistema.

IV. Dehesa cultivada. Cuando los claros son importantes aquellas zonas con mayores aptitudes agrológicas suelen ser roturadas, aunque no anualmente, y aprovechadas sus rastrojeras durante el verano por las especies domésticas: ganado bovino y ovino esencialmente. Las zonas de arbolado también serán aprovechadas para la montanera.

V. Dehesa cultivada aclarada de vuelo. Son aquellas en las que las quercíneas se reducen considerablemente en favor de las superficies roturadas quedando algunos pies de forma residual.

VI. Pastizales permanentes y superficies cultivadas. Son zonas dedicadas a cultivos leñosos (como la vid) o herbáceos. No tiene sentido denominar esta fase como dehesa, ya que desde el momento en que perdemos la presencia de uno de esos dos estratos constituyentes -suelo y vuelo- de dicho ecosistema, debemos hablar de erial o pastos, caso de pérdida de vuelo, o de monte leñoso cuan- 
do lo que se abandona es la explotación del suelo, lo que posibilita la regeneración del monte mediterráneo (LECO BERROCAL, 1996).

Dentro del sistema adehesado Llorente Pinto (1985) distingue tres estratos: estrato herbáceo y musgoso (subherbáceo), estrato arbustivo y estrato arbóreo.

- Estrato herbáceo y muscinal: está compuesto por musgos, líquenes, hongos y sobre todo, por herbáceas y pastos, fundamental en el sistema pues constituye la base alimentaria de la cabaña ganadera.

- Estrato arbustivo: este estrato es muy variable dependiendo de los diversos factores ecológicos de cada dehesa en particular y, sobre todo, de su estado de degradación. Destacan especies como el tomillo (Thymus zygis), el cantueso (Lavandula stoechas), la escoba (Cytisus multiflorus) y la retama (Lygos monosperma), la jara (Cistus ladanifer).

- Estrato arbóreo: Las especies de quercíneas más características de la dehesa son la encina (Quercus rotundifolia), el alcornoque (Quercus suber) y el rebollo (Quercus pirenaica). También se pueden encontrar el fresno (Fraxinus oxycarpa) y el acebuche (Olea europea).

\section{RECURSOS NATURALES Y APROVECHAMIENTO DE LA DEHESA.}

La explotación de la dehesa ha sido compartida, desde hace siglos, con el respeto a la naturaleza, sin alterar excesivamente el equilibrio ecológico existente. Tradicionalmente sus aprovechamientos fundamentales han sido el ganadero, el agrícola y el forestal.

Explotación ganadera. Constituye el aprovechamiento principal de la dehesa en la actualidad. La ganadería no es un elemento extraño a este ecosistema, sino que cada animal, con el consumo de la hierba al pastar, se convierte en un elemento de selección al tiempo que en un vehículo de dispersión de las semillas (que pasan a través de su aparato digestivo) y en una fuente de abono, gracias a sus excrementos.

El ganado presente en la dehesa no compite entre sí por los mismos alimentos ya que existe una complementación para aprovechar al máximo los recursos comestibles. Por un lado, el ganado bovino come los brotes de las hierbas más altas, así como también ramonea, en épocas de máxima necesidad, hasta una altura a la que no llegan los demás animales. El ganado lanar aprovecha las hierbas más cortas, previamente segadas por los bovinos. Las cabras se alimentan preferentemente de los rebrotes de los matorrales, evitando su proliferación, así como de las partes más bajas de las encinas y chaparros. Los cerdos durante la 
montanera son los principales consumidores del fruto de las encinas y los alcornoques.

Explotación agrícola. Las actividades básicas de la agricultura en la dehesa se centran en la producción de alimentos destinados al mantenimiento del ganado: producción de cereales para pienso, pastos y producción forestal. Así pues, la agricultura y la ganadería se complementan, los animales comen la hierba o pasto y, a la vez, lo abonan con sus excrementos. En los últimos años, el cultivo de cereales y de leguminosas-grano ha desaparecido prácticamente de los espacios de dehesa en Extremadura.

Explotación forestal. Se basa en el aprovechamiento de la leña y el corcho. La poda que se realiza periódicamente sobre el arbolado, necesaria para aumentar su productividad, aporta una cantidad de leña que se puede utilizar como combustible directo o para la producción de picón y carbón. La leña y madera de la encina, como tienen una salida comercial importante, ha llevado en determinadas ocasiones a podas incontroladas y salvajes, que ha provocado un avejentamiento acelerado del arbolado (aspecto que trata de atajar la Ley de Dehesas de Extremadura). De igual manera, ha resurgido la demanda de corcho gracias a sus múltiples aplicaciones: tapón para cierre de botellas, fabricación de bolsos, ropa, zapatos, etc.

Un recurso en alza es el fenómeno social, cultural y económico que supone la caza. Las actividades cinegéticas se están convirtiendo en los últimos años en una prolongación del fenómeno del ocio y del turismo, todo ello debido a los cambios culturales, mentales y de comportamiento de la relación hombrenaturaleza (LECO BERROCAL, 1996).

Otro aprovechamiento de la dehesa es su uso para el turismo rural, recurso en auge y cada vez más explotado en Extremadura.

\section{LA ENCINA: ÁRBOL REPRESENTATIVO DE LA DEHESA.}

La encina recibe el nombre científico tradicional de Quercus ilex L. Actualmente se extiende la práctica de separar sus dos subespecies principales, ilex y rotundifolia, en dos especies distintas denominadas Quercus ilex L. y Quercus rotundifolia Lam. Las dos especies mantienen en común su corteza no corchosa, el envés rugoso de sus hojas y el carácter perenne y coriáceo de las mismas. Sin embargo, se diferencian en la morfología de sus hojas y en el sabor de su fruto (bellota amarga en las Quercus ilex y dulce en las Quercus rotundifolia). Ambas reciben el nombre común de encina, aunque es frecuente también la denominación de carrasca y de chaparra. Estos dos términos se reservan sobre todo a los brotes arbustivos de raíz o al monte bajo. 


\section{Los factores físicos.}

La encina aparece preferentemente en zonas de clima mediterráneo, con inviernos moderadamente húmedos y veranos muy calurosos y secos. En lo que a temperaturas medias anuales se refiere, el rango de su distribución va desde los $8^{\circ} \mathrm{C}$ a los $19^{\circ} \mathrm{C}$, y su óptimo está comprendido entre los $10^{\circ} \mathrm{C}$ y los $18^{\circ} \mathrm{C}$. Por lo que respecta a las precipitaciones, se distribuye en un rango muy amplio, que va desde los $350 \mathrm{~mm}$ a los $1.600 \mathrm{~mm}$ anuales. Su óptimo es de entre $400 \mathrm{~mm}$ y $1200 \mathrm{~mm}$, aunque su dominio habitual está entre los 500 y los $700 \mathrm{~mm}$ anuales (MONTOYA OLIVER, 1988). La encina es capaz de soportar condiciones de sequedad casi extrema. Sobrevive en lugares donde la cantidad de precipitación anual no supera los $200 \mathrm{~mm}$ y tolera temperaturas igualmente extremas (FUENTES SÁNCHEZ, 1994).

Admite casi toda clase de suelos, incluso los rocosos y pedregosos, con las siguientes limitaciones: suelos hidromorfos, salinos y de escaso volumen útil (el que queda del accesible a las raíces una vez que se descuenta la fracción gruesa del suelo, es decir, igual o inferior a $2 \mathrm{~mm}$ ). En este tipo de suelos la encina es capaz de sobrevivir, pero no consigue alcanzar buenas dimensiones, quedando, muchas veces, reducidos sus bosques a carrascales; especialmente cuando el suelo ha sido reducido por erosión. Los suelos más óptimos para el crecimiento de la encina son aquellos que presentan características intermedias entre básicos y ácidos.

En Extremadura dominan los encinares Quercus rotundifolia Lam. Aparecen frecuentemente mezclados con alcornocal y quejigo, sobre todo en las mejores condiciones climáticas y de suelos. El cortejo de vegetación está representado por Cytisus multiflorus (escoba blanca), Lygos monosperma (retama), Quercus coccifera (coscoja), Arbutus unedo (madroño), Pistacea lentiscus (lentisco), Phillyrea angustifolia (labiérnago). En el matorral de degradación encontramos, entre otros, Cistus ladanifer (jara) y Lavandula stoechas (cantueso). En los pastizales aparecen especies como la Agrostis castellana, Poa bulbosa, Trifolium subterraneum. Degradan a las típicas "manchas", propias de la caza mayor, y en las zonas de relieve ondulado forman la mayor parte de las dehesas extremeñas. Éstas se orientan a la producción de pastos y bellotas en montanera.

\section{La poda en la encina.}

La poda es definida por el Diccionario de la Real Academia de la Lengua Española fi como "la acción y efecto de podar (cortar o quitar las ramas superfluas de los árboles, vides y otras plantas para que fructifiquen con más vigor)".

La poda puede realizarse para dar la forma adecuada al árbol o mantener la ya lograda (poda de formación), incrementar o mantener la producción de bellota 
(poda de mantenimiento), producir leña y ramón para el ganado (poda de producción) o reconstituir el follaje de árboles decrépitos (poda de rejuvenecimiento).

La poda de formación va encaminada a la consecución de ramas principales (3 ó 4) bien divergentes y limpias de ramillas que conformarán la estructura soporte de las restantes ramas de la copa. Se busca a través de ella la obtención de una ramificación equilibrada, así como aplanar y extender la copa, con el fin de abaratar la poda o mejorar la producción de bellota. La poda de formación debe efectuarse en dos tiempos, con intervalos de unos 5 ó 10 años (ha de estar precedida de una guía del tronco del árbol joven, consistente en eliminar las ramillas laterales y los chupones que salen del tronco). En la primera operación se eliminan fundamentalmente las ramas del centro, que son las más verticales: es lo que se denomina "abrir el chaparro". En la segunda operación, respetando las ramas principales elegidas, se van eliminando los brotes verticales.

El fundamento de la poda de mantenimiento es aumentar la fructificación, reduciendo al mínimo el ángulo que forma la rama con el horizonte del suelo de modo que la savia elaborada descienda más lentamente. Si se eliminan las ramas secas o interiores, así como las verticales, que son menos fructíferas, la savia bruta que recibían pasa a las ramas horizontales y colgantes que producirán y retendrán más savia elaborada y, con ello, más fruto, motivando también la ampliación de la copa (DELGADO GIL, 1984). En general, esta poda debe realizarse tras la formación del árbol y cuando éste tiene edad y tamaño para producir bellota adecuadamente, a partir de unos 50 años (aproximadamente $75 \mathrm{~cm}$ de circunferencia a 1,30 m del suelo). Se caracteriza por su moderación, tanto en cuantía como en grosor de ramas cortadas (no mayores de 4-5 cm de diámetro) (MONTOYA OliVer, 1988).

La poda de producción es más agresiva para el árbol que la anterior. Con ella se pretende conseguir más leña y más gruesa, con lo que el árbol y la fructificación salen perjudicados. Se puede justificar, hasta cierto punto, solamente cuando la producción de bellota interesa menos que la producción de leña, o porque los costes de la poda de mantenimiento obliguen a espaciarla en el tiempo y a efectuar una poda más dura para compensar gastos (DELGADO GIL, 1984).

La poda de rejuvenecimiento, en teoría, se efectúa en árboles envejecidos, con ramas que empiezan a perder brotes y a secarse por los extremos superiores de la copa. Al eliminar buena parte de las ramas, a niveles próximos al tronco, el árbol reacciona ante la poda (si es capaz de aguantar) y produce una maraña de jóvenes chupones que dan una impresión de rejuvenecimiento. Esta técnica es perjudicial para las encinas, porque estos árboles en situación de precario crecimiento serán incapaces de cicatrizar los cortes producidos, con lo cual su lon- 
gevidad se verá acortada. El gran problema de este tipo de poda es que se realiza sobre árboles de cualquier edad, y no sólo sobre los envejecidos, por lo que se constituye como la poda más abusiva.

Aparte de estas podas artificiales existe una poda natural, que como su propio nombre indica, la realiza el árbol sin la intervención del hombre. Se rige por el principio de lucha por la luz. En la encina se produce fundamentalmente en ramas interiores, aunque también en ramas inferiores. Conforme las ramas, por la circunstancia que sea (crecimiento bajo, lesión, etc.), pierden su salida a la luz, eclipsadas por sus competidoras, van declinando su crecimiento hasta secarse, por lo que con el tiempo se quiebran y son de este modo eliminadas. La poda por el hombre de estas ramas interiores secas es, en realidad, seminatural y es la poda más beneficiosa para el árbol (aunque la menos provechosa para el podador).

Las podas deben realizarse con una periodicidad de 6 a 12 años, pues pasada ya la crisis de la anterior poda, el árbol ha reconstituido su follaje, tiene ya buen crecimiento diamétrico y, consecuentemente, una buena capacidad de cicatrización. Retrasar en exceso las podas permite engrosar excesivamente el ramaje que debería ser cortado y que acaba por deformar definitivamente el porte del árbol.

La época ideal es el período de reposo vegetativo, por paralizarse al máximo la circulación de la savia, así ni se pierde ésta ni se desprende la corteza. Suele hacerse desde octubre, si no tiene bellota, hasta mediados de marzo, aunque su óptimo es desde primeros de diciembre a primeros de marzo.

\section{La encina en la dehesa.}

La dehesa, como paisaje a medio camino entre el bosque primitivo mediterráneo y el pastizal desarbolado o la explotación extensiva de cereal, permite la convivencia de estructuras vegetales simples, como el pasto, y otras más maduras, como los árboles. La supervivencia de estas últimas es fundamental para que el funcionamiento del sistema no sufra cambios irreparables (LLORENTE PINTO, 1985).

La influencia del arbolado es decisiva en los paisajes adehesados. La encina, como árbol representativo de la dehesa, desempeña un papel fundamental en el funcionamiento del sistema. Se pueden distinguir tres aspectos en los que el árbol ejerce su influencia sobre el ecosistema dehesa: sobre el suelo, la vegetación y la distribución del agua de lluvia.

Con sus raíces, aferradas a unos suelos pobres, reduce los efectos de la erosión, a la vez que ejerce una acción de bombeo de la humedad y de otros nu- 
trientes desde el subsuelo hacia la superficie, que, junto con los aportes de materia orgánica y humus (los animales buscan la sombra de los árboles para refugiarse y descansar, y con sus excrementos fertilizan el suelo), favorece un enriquecimiento del mismo.

Fuera de la proyección de la copa, la influencia sobre el suelo es muy escasa (en especial en la encina) y bajo la proyección se pueden distinguir dos sectores: uno de fuerte influencia, correspondiente a la parte más septentrional, donde el efecto de la sombra es más constante lo que favorece la humificación del suelo, y otro en el que la influencia es más débil, situado al sur del tronco (LLORENTE PINTO, 1985).

La vegetación es más abundante y su longevidad es mayor bajo la copa de las encinas porque los contenidos de materia orgánica, humus y humedad son mayores. Además, la copa del árbol actúa de pantalla frente a la acción del viento, las heladas y la radiación solar, protegiendo de esta manera la vegetación. Así, las condiciones microclimáticas creadas por el arbolado favorecen una germinación más temprana, un mayor crecimiento en el período frío, un retraso en la floración y un agostamiento más tardío.

La pantalla que constituye la copa de los árboles intercepta parte de las precipitaciones y provoca una desigual distribución, sobre el suelo, del agua que pasa a través de ella. La influencia de la copa es diferente para toda su área de proyección, así se pueden establecer tres zonas: la zona de influencia inmediata, que es la más próxima al tronco y abarca un diámetro de aproximadamente $1 \mathrm{~m}$; la zona de influencia de la copa, con un diámetro de unos $15 \mathrm{~m}$ y donde los efectos beneficiosos de la cubierta son máximos; y la zona de influencia alejada, que puede alcanzar hasta los $100 \mathrm{~m}$ y donde el efecto de la copa va disminuyendo (PENCO MARTíN, 1992).

También la encina juega un papel importante como soporte físico en donde nidifican gran número de aves (cigüeñas o urracas), viven numerosos mamíferos en los huecos de sus ramas (jinetas o lirones), o se refugian al amparo de su sombra tanto animales salvajes (ciervos o jabalíes), como domésticos (ovejas o vacas).

Además de todo esto, no hay que olvidar la fuente de alimentos que supone su fruto (sobre todo la bellota) para los animales que habitan en el ecosistema dehesa; así como tampoco, los beneficios, tanto económicos como silvopastoriles, que aporta la poda (tratada en el apartado anterior).

Podemos resumir las funciones ecológicas, silvícolas y pastoriles del arbolado en cinco aspectos: estabilización de la producción; mejora de las condiciones de estanco para el ganado: protección ante el frío, el sol, la lluvia, el viento, etc.; 
aportaciones de los beneficios propios del árbol: alimento (bellota y ramón), leña, protección del suelo frente a la erosión, además de los beneficios ecológico generales asociados a la presencia del arbolado (conservación de la fauna, mejora del paisaje, etc.); simplificación de la ordenación forestal: el pasto bajo la copa se convierte en una reserva forrajera natural desde la primavera hasta la llegada de la sequía estival; inversiones y mejoras silvopastoril: un ejemplo lo constituye la poda.

\section{DISTRIBUCIÓN DE LAS QUERCÍNEAS EN EXTREMADURA.}

Los espacios de dehesa se localizan por toda la zona mediterránea (fundamentalmente en la parte mediterránea occidental). España es el primer país en cuanto a extensión del dominio de la encina, siendo la primera especie, en superficie ocupada, dentro de nuestros árboles forestales (MONTOYA OLIVER y OTROS, 1988), a pesar de la creciente destrucción del bosque.

Aparece en casi todas las provincias de España, llegando a ocupar una superficie de 2.889.341 ha, el 24\% del área arbolada de nuestro país (Moro, 1995). Se presenta de manera abundante en la zona centro y meridional de la Península (Extremadura, Salamanca y Andalucía) y en menor proporción en el norte (Galicia, Asturias y Guipúzcoa, donde el clima no es adecuado para su crecimiento) y el este peninsular (Cataluña, Valencia, Murcia y Baleares), donde ha sido prácticamente destruida y sólo quedan carrascales y pies dispersos.

Mientras la Quercus ilex se extiende por el norte y este peninsular y Baleares, la Quercus rotundifolia lam lo hace por el centro, Oeste y sur peninsular.

En Extremadura la superficie que ocupan las quercíneas supera el millón de ha, si bien, según las fuentes consultadas y la nomenclatura de clasificación de las mismas, los datos referentes a la superficie ocupada sufren pequeñas modificaciones.

De esta manera, según LECO BERROCAL (1995), las quercíneas ocupan en Extremadura una superficie de $1.211 .883 \mathrm{ha}$, 1o que supone aproximadamente un $29,3 \%$ del total de la superficie regional.

Según los datos del Proyecto CORINE Land Cover (CLC, 2000) la superficie ocupada asciende a 1.129.352 ha de arbolado de quercíneas y 501.168 ha de matorral arbolado (Ver MAPA 1).

Por otra parte, el Mapa Forestal de España (2000), escala 1:50.000, estima la superficie ocupada por quercíneas en 1.390 .556 ha. En todo caso, estas cifras nos ponen de manifiesto la importancia de la superficie arbolada de quercíneas en la región. Y, especialmente, del encinar que ocupa, según los datos del MFE, 
un total de 1.142 .049 ha en masas puras y 162.889 ha en formación mixta junto con otras quercíneas, todo ello suponen un total de 1.304 .938 ha, un $94 \%$ sobre el total de quercíneas. El encinar ocupa, además, el 3 1,3\% de la superficie regional.

Cuadro 1. Distribución de las quercíneas en Extremadura

\begin{tabular}{lrrrrr}
\hline \multicolumn{1}{c}{ Provincias } & Encinar & Alcornocal & Encinar Mixto & Rebollar & Total \\
\hline Badajoz & 637.496 & 17.825 & 71.743 & 523 & 727.587 \\
Cáceres & 504.553 & 43.273 & 91.146 & 23.996 & 662.968 \\
Extremadura & 1.142 .049 & 61.098 & 162.889 & 24.519 & 1.390 .55 \\
\hline
\end{tabular}

FUENTE: Elaboración propia a partir del MFE, 1:50000

\section{BIBLIOGRAFÍA}

Bravo Oviedo, F. (1989): «Estudio silvopastoril de la Dehesa Boyal de Alía (Cáceres)». Ecología, 3. ICONA, Madrid, pp. 107-115.

CAMPOS PALACÍN, P. (1992): «Reunión Internacional sobre sistemas agroforestales de dehesas y montados». Agricultura y Sociedad, 62. MAPA, Madrid, 197-202.

DELGADO GIL, A. M. (1984): «Las podas, un factor de destrucción del encinar». Quercus, 15, pp16-19.

ELENA Roselló, M. (1986): «Tipología de explotaciones de dehesa en Extremadura. Una aplicación del ACP». 1 Jornadas Técnicas sobre Conservación y Desarrollo de las Dehesas Portuguesa y Española. MAPA, Madrid, pp. 469-486.

FUENTES SÁNCHEZ, C. (1994): La encina en el centro y suroeste de España (su aprovechamiento y el de su entorno). Junta de Castilla y León, Consejería de Medio Ambiente y Ordenación del Territorio. Castilla y León.

HERNÁNDEZ DíAZ-AMBRONA, C. G. (1995): «La dehesa extremeña: Un entorno claramente hostil». Agricultura, 750, pp. 37-41.

LECO BERROCAL, F. (1995): Ecosistema dehesa. Aproximación metodológica a su delimitación, ordenación y transformación. Tesis Doctoral. Facultad de Filosofía y Letras, Dpto. de Geografía y O.T., UEX, Cáceres.

LECO BerRoCAl, E. (1996): Delimitación y modelización de los espacios adehesados extremeños. Dpto. de Geografía y O.T., UEX, FUNDICOTExtremadura y Consejería de Cultura y Patrimonio de la Junta de Extremadura, Cáceres, 268 pp.

LEy De DeHESAs En EXTREMADURA De 1/1986, de 2 de mayo (D.O.E., $n^{0} 40$, de 15 de mayo de 1986).

LlORENTE PINTO, J. M. (1985): Los paisajes adehesados salmantinos. Centro de Estudios Salmantinos, Salamanca. 
Montoya Oliver, J. M. (1988): Encinas y encinares. Ed. Mundi-Prensa, Madrid.

Montoya Oliver, J. M.; Mesón García, M. L. y Ruiz CAstillo, J. (1988): Una dehesa testigo. La dehesa de Moncalvillo. Serie Técnica, $\mathrm{n}^{\circ}$ 5. ICONA, Madrid.

Moro, R. (1995): Guía de los árboles de España. Ed. Omega, Barcelona.

Penco Martín, A. D. (1992): Aproximación a la dehesa extremeña. Diputación Provincial de Badajoz, Badajoz.

SOCIEDAD ESPAÑOla PARA El EstUdio De LOS PASTOS (2001): Nomenclátor básico de pastos en España. Pastos: Revista de la Sociedad Española para el Estudio de los Pastos, vol. XXXI. 


\section{OCUPACIÓN DE LOS ESPACIOS ADEHESADOS EXTREMEÑOS}

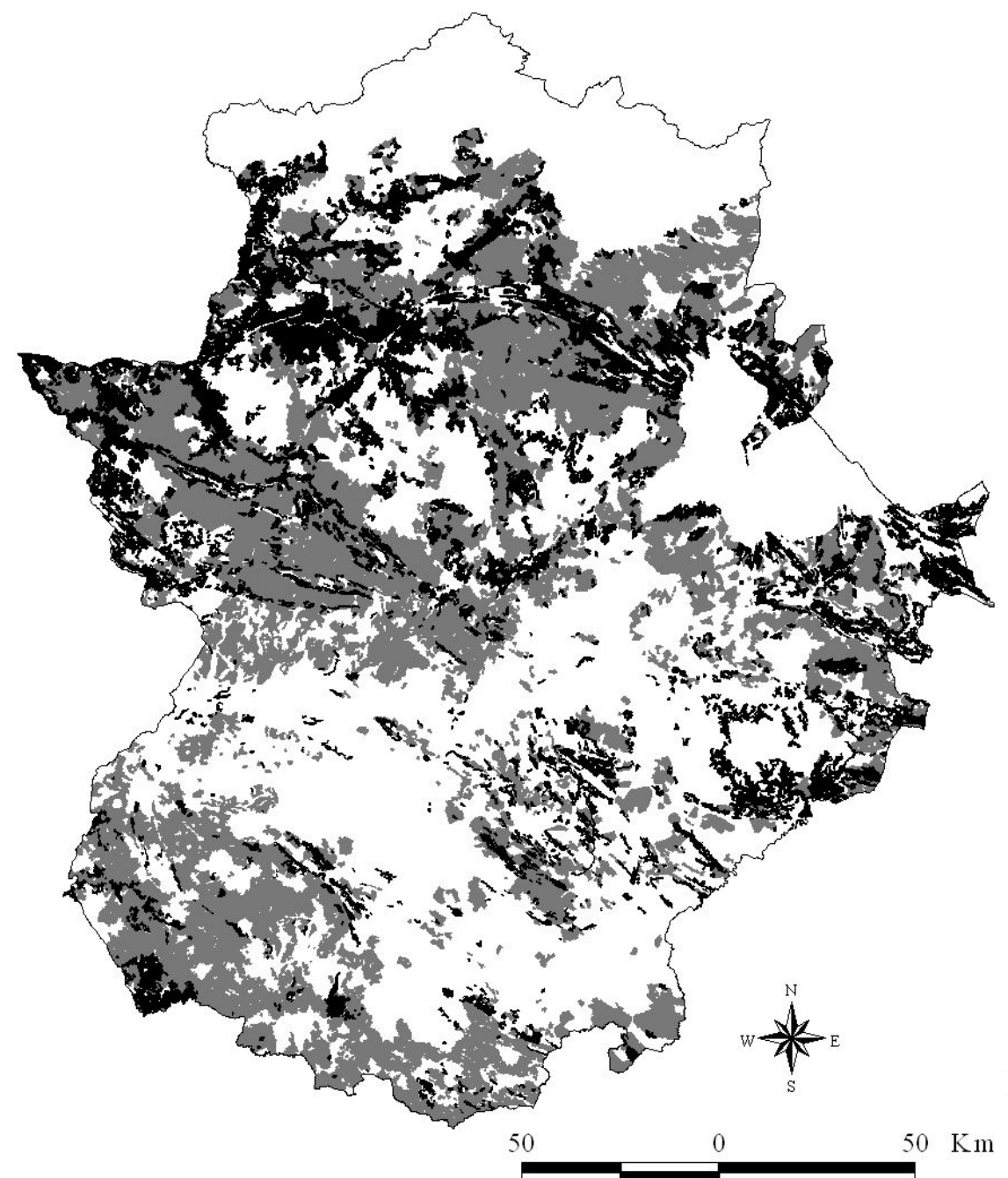

\section{LEYENDA}

Arbolado

Formaciones de matorral
(Fuente: Elaboración propia a partir de la información contenida en el proyecto CORINE Land Cover, 2000) 